\title{
Tiv Language
}

National Cancer Institute

\section{Source}

National Cancer Institute. Tiv Language. NCI Thesaurus. Code C154168.

A Niger-Congo Southern Bantoid language spoken in Nigeria, mainly in the Benue State. 